\title{
Psicooncología
}

ISSN: 1696-7240

\section{Influencia del apoyo social sobre el estrés y la satisfacción vital en padres de niños con cáncer desde una perspectiva multidimensional}

\author{
Anabel Melguizo-Garín ${ }^{1 *}$; Mª José Martos-Méndez²; Isabel Hombrados-Mendieta ${ }^{3}$
}

Recibido el 22 de febrero de 2019 / Aceptado el 7 de marzo de 2019

Resumen. Objetivo: El presente estudio se plantea como objetivo principal analizar cómo la percepción de apoyo de los padres de niños diagnosticados con enfermedad oncológica influye en la percepción de estrés y la satisfacción vital. Método: Participaron 112 padres de niños con patología oncológica que recibían tratamiento en Málaga y pertenecían a una asociación de padres de niños afectados por esta enfermedad. Resultados: Respecto a las fuentes analizadas la percepción de apoyo familiar disminuye la frecuencia del estrés, el apoyo de los amigos y de las asociaciones disminuye el esfuerzo relacionado con el estrés y el apoyo de la pareja aumenta la satisfacción vital de los progenitores. Respecto a los tipos de apoyo, el apoyo instrumental es el que más reduce la frecuencia del estrés de los progenitores, el apoyo informacional reduce el estrés relacionado con el esfuerzo que los padres deben realizar y es la satisfacción con el apoyo emocional la variable que mejor predice la satisfacción vital. Conclusión: El análisis multidimensional del apoyo social ha permitido analizar con mayor profundidad la complejidad de las relaciones de apoyo social que acontecen durante la enfermedad oncológica de los hijos. Del estudio se derivan importantes implicaciones prácticas.

Palabras clave: padres de niños con cáncer, apoyo social, estrés, satisfacción vital.

\section{[en] Influence of social support in stress and life quality in parents whose children have cancer from a multidimensional perspective}

\begin{abstract}
Objective: To analyse how the social support of parents with children diagnosed with oncological disease influences stress perception and quality of life. Method: 112 parents whose children suffer oncological pathologies received treatment in Malaga. They belonged to an association of parents with children affected by this disease. Results: According to sources used, perception of family support decreases the stress frequency, friends support is also an important aid alleviating the stress. In addition, support from a partner improves parents' well-being. There are different types of support: firstly, instrumental support, which reduces the frequency of parents' stress; secondly, informational
\end{abstract}

1 Anabel Melguizo-Garín Departamento de Personalidad, Evaluación y Tratamiento Psicológico. Facultad de Psicologia. Universidad de Malaga. España.

E-mail: anamel@uma.es

2 Ma José Martos-Méndez. Departamento de Psicologia Social. Facultad de Psicologia. Universidad de Malaga. España.

E-mail:mjmartos@uma.es

3 Isabel Hombrados-Mendieta. Departamento de Psicologia Social. Facultad de Psicologia. Universidad de Malaga. España.

E-mail: mihombrados@uma.es

* Dirección de correspondencia: Anabel Melguizo-Garín. Departamento de Personalidad, Evaluación y Tratamiento Psicológico. Facultad de Psicología. Universidad de Malaga. Campus Universitario de Teatinos s/n. 29071 Malaga (Spain). E-mail: anamel@uma.es. 
support reducing stress related to the effort that parents must exert; thirdly, the most important variable to get quality of life is emotional support. To conclude: The multidimensional analysis of social support allows us to analyse the complexity of social support taking place during oncological illnesses in children. Practical implications come as a result from this study.

Keywords: parents whose children have cancer, social support, stress, quality of life.

Sumario. 1. Introducción 2. El contexto familiar 3. Repercusión del estrés en los padres de niños con cáncer 4. Importancia del apoyo social como factor protector ante el estrés 5. La satisfacción vital de los padres de niños con cáncer 6. Objetivos 7. Método 8. Resultados 9. Discusión y conclusiones 10. Agradecimientos 11. Referencias bibliográficas.

Cómo citar: Melguizo-Garín A, Martos-Méndez MJ, Hombrados-Mendieta, I. Influencia del apoyo social sobre el estrés y la satisfacción vital en padres de niños con cáncer desde una perspectiva multidimensional. Psicooncología 2019; 16(1):25-42. doi: 10.5209/PSIC.63646.

\section{Introducción}

El cáncer infantil es una enfermedad que cambia por completo la vida del menor afectado, así como la de sus familiares y entorno más cercano. En el momento en el que el niño es diagnosticado de cáncer no sólo cambia su estado de salud, si no que sus rutinas, su vida académica, social y familiar se ven afectadas. El cáncer irrumpe en la vida de las familias con todo su bagaje vital, psicológico y social ${ }^{(1)}$. Además de los cambios que se producen en el menor, la vida de sus progenitores sufre un desajuste, ya que las rutinas y la vida diaria de los padres tienen que adaptarse a la nueva situación. Un cáncer es un elemento desestabilizador para cualquier familia ${ }^{(2)}$. Los cambios en estas rutinas de la vida diaria, así como el afrontamiento de situaciones novedosas y hasta ahora desconocidas para los padres, como por ejemplo las largas permanencias en el centro hospitalario ${ }^{(3)}$, pueden generarles estrés y niveles elevados de esfuerzo para poder continuar con la vida familiar a la vez que se ha de cuidar del hijo enfermo.

El diagnóstico de un cáncer infantil supone un desafío para las familias que deben enfrentarse a diferentes momentos de crisis, en palabras de López-Ibor ${ }^{(1)}$ "todo comienza el día del diagnóstico, cuando todo parece que se derrumba". El diagnóstico, tratamiento, incorporación a la vida escolar, familiar y social, posibilidad de recidivas $\mathrm{y}$, en algunas ocasiones, el fallecimiento del niño o niña con las graves consecuencias emocionales derivadas, son situaciones que pueden desencadenar momentos de crisis en los progenitores y en toda la familia del menor enfermo ${ }^{(4,5)}$. Los tratamientos frecuentes, las hospitalizaciones, los efectos secundarios y el miedo a la recaída pueden contribuir a un aumento del estrés por parte de los padres ${ }^{(6)}$, esta sintomatología, en ocasiones, puede persistir incluso una vez finalizado el tratamiento del hijo ${ }^{(7,8)}$. No obstante, parece que existen algunos factores de protección por los cuales no todos los padres experimentan los mismos efectos psicológicos a lo largo del tiempo ${ }^{(9)}$.

\section{El contexto familiar}

El entorno de las personas o el contexto social en el que viven, además de poder ser una fuente importante de apoyo, también puede considerarse, en algunas ocasiones, 
un factor de estrés para el individuo ${ }^{(10)}$, que generará una serie de respuestas para estos estresores. Los niños y sus familias forman parte de distintos sistemas, que a su vez interactúan entre ellos (familias extensas, escuelas, actividades de ocio, sistema sanitario, trabajo, etc.), por tanto, ante el diagnóstico de la enfermedad en el niño, los distintos sistemas se activarán y reaccionarán de diferente modo ${ }^{(11)}$. El modelo ecológico de Bronfenbrenner sugeriría que los recursos ambientales o sociales pueden mitigar la respuesta psicológica asociada con la experiencia de tener un hijo con cáncer. El análisis del modelo ecológico aplicado a la psicooncología destaca la importancia del apoyo social que recibe el paciente oncológico proveniente de diferentes entornos ${ }^{(12)}$. Lo original e interesante de la aplicación de este modelo en pacientes con cáncer es que se tiene en cuenta el "ambiente ecológico" que circunscribe al individuo, considerando a éste como un sujeto en progresiva acomodación a sus entornos inmediatos y cambiantes, y a su vez dichos entornos se van adaptando al sujeto $^{(10)}$. El modelo ecológico de Bronfenbrenner ${ }^{(10)}$, ha sido ampliamente estudiado y extrapolado a diferentes ámbitos como el educativo, sociofamiliar y de salud. Sus postulados pueden servir como paradigma y marco teórico para diferentes contextos y disciplinas dentro de la psicología, por lo que estos supuestos pueden ser trasladados a la situación contextual de los padres con hijos con cáncer. Debido al hecho traumático de la enfermedad de uno de sus miembros de la familia, es toda ella en cierto modo la que "enferma" (13), y pone en marcha estrategias para el afrontamiento de la enfermedad en las que el contexto familiar juega un papel relevante ${ }^{(14,15)}$.

\section{Repercusión del estrés en los padres de niños con cáncer}

Los padres pueden encontrarse con diferentes situaciones estresantes: a) relacionadas con la enfermedad del hijo, como efectos secundarios a los tratamientos de radioterapia y quimioterapia, déficits atencionales y cognitivos, cansancio y fatiga, caída del cabello, debilitamiento físico y emocional, miedos y frustraciones del hijo; b) problemas familiares que acarrean sensación de pérdida de control, miedos e incertidumbres con respecto a la enfermedad del hijo, culpa, pensamientos invasivos, dificultad para encontrar el equilibrio en las necesidades de la familia, falta de comunicación, conflictos con la familia extensa, conflictos con los otros hijos, problemas de pareja, financieros, etc.; c) situaciones de distanciamiento por parte de amistades, ausencia de apoyo de las instituciones, falta de ayudas económicas y fallecimientos de otros niños; d) situaciones relacionadas con el sistema sanitario que pueden generar estrés en los padres como es la comunicación pobre o errónea de información, retrasos en los diagnósticos y las pruebas, toma de decisiones precipitadas y largas estancias en hospitales poco equipados para los familiares ${ }^{(16,17)}$.

Todas las situaciones anteriores requieren de esfuerzo por parte de los padres para poder hacerles frente y uno de los modos para reducir esos elevados niveles de estrés es la petición de ayuda a la pareja, los familiares y el entorno cercano ${ }^{(18)}$. Los progenitores tienen que desempeñar tareas complejas de cuidado que requieren gran esfuerzo, viviendo una situación complicada de gestionar, tomando decisiones difíciles sobre la situación del hijo enfermo. Todo esto puede dar lugar a que la vida de pareja, familiar y social se vea resentida y también sufra un desajuste que, en 
muchas ocasiones, lleva a desencuentros y percepción de falta de apoyo, que se unen a la situación estresante y de crisis que ya está viviendo la familia debido al cáncer infantil. En estos casos, el apoyo que reciben las madres y los padres puede ser de gran ayuda para paliar las posibles dificultades con las que se encuentran para poder desarrollar todas las labores y funciones que han de afrontar. La cohesión entre los miembros de la familia, así como una adaptación positiva a la situación se relacionan negativamente con el estrés que experimentan los padres de niños con cáncer ${ }^{(6,19)}$.

\section{Importancia del apoyo social como factor protector ante el estrés}

El apoyo social es una de las variables que más en cuenta se ha tenido en las investigaciones sobre psicooncología, ya que es importante a la hora de determinar cómo afrontará el paciente oncológico su enfermedad ${ }^{(20)}$, entendiendo que el apoyo social puede influir en la calidad de vida y el estrés ${ }^{(21)}$. Así, en pacientes con cáncer, el apoyo social es altamente beneficioso, ya que suele disminuir los niveles de estrés que éste experimenta ${ }^{(22)}$. El apoyo social es una de las áreas de investigación que más se ha estudiado en las últimas décadas ${ }^{(23,24)}$ y puede ser analizado desde la perspectiva de las distintas fuentes y los diferentes contextos de apoyo, como indican los modelos ecológicos y sistémicos ${ }^{(25)}$. El apoyo social es un concepto interactivo, es una transacción interpersonal de ayuda que se produce entre las fuentes de apoyo y el receptor de la ayuda, que puede implicar emociones, ayuda material e información y que se da en un contexto determinado ${ }^{(26)}$. El apoyo social se define como los recursos sociales que las personas perciben que tienen disponibles ${ }^{(27)}$ y que les son proporcionados de manera formal por contextos o grupos profesionales $\mathrm{o}$ instituciones y asociaciones, o de manera informal por grupos de apoyo como familia, amigos y pareja. En resumen, el apoyo social se refiere a la percepción o la experiencia de que uno es amado y cuidado, estimado y valorado, y es parte de una red social de asistencia mutua ${ }^{(28,29)}$.

Entre los recursos que los padres utilizan para hacer frente al estrés se encuentra el apoyo de la familia extensa, el apoyo de la comunidad, las asociaciones, apoyos religiosos, la escuela, redes sociales de afectados por la enfermedad, etc. ${ }^{(30)}$. El cáncer de un niño tiene una repercusión social importante y esto promueve la creación de asociaciones y entidades para atender las necesidades psicosociales de los padres ${ }^{(1)}$. Otros estudios también apuntan a que el apoyo social se relaciona con niveles de estrés más bajos, y facilita la adaptación ${ }^{(31,32)}$. En este sentido Espada y $\mathrm{Grau}^{(14)}$ describieron que la búsqueda de apoyo social por parte de los padres de niños con cáncer es una de las estrategias de afrontamiento más utilizadas por los mismos, en este estudio las principales fuentes de apoyo descritas son el apoyo proporcionado por el personal sanitario, el apoyo de pareja, de la familia extensa, amigos y espiritual.

Debido al tratamiento al que habitualmente se someten los niños enfermos de cáncer, la familia necesita contar en muchas ocasiones con apoyos externos al núcleo familiar. Suele producirse la entrada de otros miembros de la familia más extensa en la toma de decisiones y en la asunción de roles del núcleo familiar, como los abuelos, los tíos, etc. La familia puede ser una fuente muy importante de apoyo a los padres $^{(31)}$. Disponer de personas de confianza a las que poder expresar emociones, problemas o dificultades, escuchar su opinión, o simplemente tener la sensación de 
ser escuchados y aceptados como personas, ha demostrado tener un fuerte impacto en la capacidad de los individuos para afrontar adecuadamente situaciones difíciles y estresantes ${ }^{(33)}$, por lo que el apoyo social podría funcionar como un protector ante la aparición de problemas y situaciones estresantes. La satisfacción con el apoyo social puede atenuar el estrés y la ansiedad de los padres de niños con cáncer y, además, puede ser un factor de protección ante el desarrollo de trastornos psicológicos en el futuro y, por tanto, para la salud de los padres ${ }^{(34)}$.

\section{La satisfacción vital de los padres de niños con cáncer}

La enfermedad oncológica también puede repercutir en la satisfacción vital de los padres. La calidad de vida es una de las variables que más se ve afectada por la enfermedad. El cuidado de un hijo enfermo de cáncer puede influir en la baja satisfacción vital de las madres que asumen el papel de cuidadora principal ${ }^{(14)}$. El progenitor que asume el rol de cuidador principal pone en marcha una serie de estrategias para procurar el bienestar del hijo enfermo, manteniendo una interdependencia emocional con éste, manejando la información que recibe y contribuyendo a una actitud de colaboración con los tratamientos ${ }^{(14)}$, estas obligaciones pueden poner en peligro la relación con los otros hijos ${ }^{(35)}$. Los hermanos del niño enfermo son los grandes olvidados ${ }^{(36)}$, los padres sometidos a las exigencias de los tratamientos del hijo enfermo se ven obligados a relegar a un segundo plano las necesidades de los hijos sanos ${ }^{(36)}$, este hecho puede ser una fuente de estrés e insatisfacción vital. Los padres se tienen que enfrentar a múltiples situaciones que les generan estrés ${ }^{(37)}$, lo que puede incidir de manera negativa en su calidad de vida $\mathrm{y}$ han de poner en marcha recursos para enfrentarse a dichas situaciones. El apoyo social es un recurso valioso para los padres de niños con cáncer, desempeñando éste un papel positivo en la satisfacción vital ${ }^{(38-40)}$. La mejora de la calidad de vida del niño enfermo de cáncer y de su familia necesita del tratamiento de los aspectos psicosociales $^{(36)}$.

\section{Objetivos}

El presente estudio se plantea como objetivo principal analizar cómo la percepción de apoyo de los padres de niños diagnosticados con enfermedad oncológica influye en la percepción de estrés y la satisfacción vital.

Una aportación importante de la investigación es que se realiza un análisis multidimensional del apoyo social. Este análisis multidimensional permitirá diferenciar entre los tipos de apoyo (emocional, instrumental e informacional) y las fuentes que lo proporcionan (pareja, familia, amigos y comunidad/asociaciones). A pesar de que el apoyo social debe ser analizado desde la perspectiva de las distintas fuentes y los distintos tipos de apoyo son muy escasos los estudios que en este ámbito lo realizan. También son escasos los trabajos que analizan el estrés atendiendo a su doble dimensión (frecuencia y esfuerzo). La dimensión frecuencia hace referencia al número de ocasiones que los padres tienen que afrontar situaciones potencialmente estresantes relacionadas con la enfermedad del niño y la dimensión esfuerzo se refiere a la carga que les supone enfrentar esas situaciones. 
De acuerdo con los objetivos del estudio las hipótesis que se plantean son:

1. Esperamos encontrar una relación positiva entre apoyo social y satisfacción vital y una relación negativa entre apoyo social y estrés percibido por los padres.

2. La percepción de apoyo social (emocional, instrumental e informacional) que proporcionan las diferentes fuentes (pareja, familia, amigos y comunidad/ asociaciones) reducirá el estrés percibido de los padres en su doble dimensión (frecuencia y esfuerzo).

3. La percepción de apoyo social (emocional, instrumental e informacional) que proporcionan las diferentes fuentes (pareja, familia, amigos y comunidad/ asociaciones) aumentará la satisfacción vital de los padres.

\section{Método}

\section{Participantes}

Participaron en este estudio 112 padres de niños con patología oncológica que recibían tratamiento en Málaga. La selección de los participantes se realizó contando con todos los padres que quisieron participar en el estudio y pertenecían a una asociación de padres de niños afectados por esta enfermedad. Los participantes se encontraban en diferentes fases de la enfermedad del hijo y del tratamiento. La muestra estaba formada por $33,9 \%$ hombres y $66,1 \%$ mujeres, con una media de edad de 41 años $(\mathrm{DT}=6,93)$. Los padres participantes en el estudio, en su mayoría, estaban casados o vivían en pareja $88,5 \%$, estando el resto solteros, divorciados o viudos $11,5 \%$. En cuanto al sexo de los niños que padecían la enfermedad, 58\% eran niños y $42 \%$ niñas, y la media de edad fue de $8(\mathrm{DT}=5,02)$. Con respecto al tipo de cáncer, el 54,5\% padecían leucemia, el 9\% sarcoma de Ewing, el 8\% linfoma, el 4,5\% meduloblastoma, el 4,5\% neuroblastoma, y el resto, otros tipos de patologías oncológicas. Por último, el tiempo transcurrido desde el diagnóstico de la enfermedad ha sido el siguiente: el $18,9 \%$ de los niños han sido diagnosticados hace menos de un año, el 23,4\% hace un año, el 19,8\% hace dos años, el 9,9\% hace tres años, el $12,6 \%$ hace 4 años y el resto $15,3 \%$ hace cinco o más años desde el diagnóstico de la enfermedad.

\section{Procedimiento}

Los padres participantes eran usuarios o habían estado en alguna ocasión en contacto con una ONG local que trabaja prestando apoyo a familias con niños con cáncer. Se informó a los padres registrados en la ONG del estudio. Los participantes recibieron un consentimiento informado sobre el procedimiento que se llevaría a cabo. Es importante resaltar que este estudio cuenta con la aprobación del Comité Ético de investigaciones científicas de la Junta de Andalucía (España), CEI 2017. Los padres participantes tras la firma del consentimiento informado pudieron elegir dos formas de completar el instrumento: en papel en las dependencias de la ONG, o en formato on-line a través de un modelo de instrumento informatizado que una vez recogido se añadía de manera anónima y automática a una base de datos. 


\section{Instrumentos}

Los instrumentos utilizados para medir las variables del estudio fueron los siguientes:

Cuestionario sociodemográfico en el que se pregunta por datos relevantes para la investigación: sexo, edad, estado civil. También se pregunta por el hijo enfermo: sexo, tipo de cáncer, tiempo del diagnóstico.

Inventario Pediátrico para Padres (PIP), versión española del Pediatric Inventory for Parents de Streisand et al. (2001) adaptación y validación de Del Rincón, Remor y Arranz (2007) $)^{(41)}$. Este cuestionario evalúa el estrés que generan las situaciones a las que se enfrentan diariamente los padres de niños con enfermedad oncológica. Se compone de una escala de frecuencia y otra escala de esfuerzo. Cada una de las escalas consta de 42 preguntas relacionadas con situaciones a las que los padres han de enfrentarse durante la enfermedad del hijo, éstos tienen que contestar en primer lugar con qué frecuencia ocurre dicho ítem pudiendo elegir entre cinco opciones de respuesta en la que " 1 " es nunca y " 5 " es muy a menudo; y cuánto esfuerzo le supone dicha afirmación, eligiendo entre 5 opciones en la que " 1 " es nada y " 5 " es muchísimo.

Cuestionario de Frecuencia y Satisfacción con el Apoyo Social (QFSSS) de García-Martín, Hombrados-Mendieta y Gómez-Jacinto $(2016)^{(42)}$. Este cuestionario se utilizó para medir la percepción de apoyo social de los progenitores, en concreto, el tipo de apoyo (emocional, instrumental e informativo) que prestan cada una de las fuentes de apoyo provenientes de la red social de los padres (pareja, familia, amigos y miembros de la comunidad y asociaciones). Por tanto, está compuesto por un total de 12 ítems. Las opciones de respuesta para cada dimensión son cinco, siendo en la dimensión frecuencia "1" nunca y " 5 " siempre, y en la dimensión satisfacción "1" insatisfecho" y "5" muy satisfecho.

Escala de satisfacción con la vida de Diener et al.(43). Esta escala ofrece un índice general de satisfacción con la vida, entendida ésta como un constructo general de bienestar subjetivo. Es una escala unidimensional compuesta por 5 ítems que se responden con una escala tipo Likert de 7 puntos $(1=$ completamente en desacuerdo y $7=$ completamente de acuerdo).

\section{Resultados}

Para conocer el nivel de estrés que padecen los progenitores, su satisfacción vital, así como la satisfacción con el apoyo social recibido, se han realizado una serie de análisis descriptivos que pueden verse en la tabla 1 . Los resultados indican que los padres manifiestan sentir un estrés medio-alto cuando tienen que hacer frente a las situaciones relacionadas con la enfermedad que padecen sus hijos. En concreto, les produce más estrés la frecuencia o el número elevado de situaciones de este tipo que el propio esfuerzo que les lleva realizar estas actividades. Por otro lado, los padres indican recibir un apoyo medio de las distintas fuentes de apoyo social analizadas. Además, la satisfacción con los distintos tipos de apoyo recibidos es también media. Los padres indican tener un nivel medio de satisfacción vital, esto es, no se encuentran del todo satisfechos con su vida. En la tabla 1, puede observarse el nivel de fiabilidad de cada uno de los instrumentos utilizados en el estudio, calculado a través del alfa de Cronbach. 
Tabla 1: Nivel de satisfacción vital, estrés y apoyo social

\begin{tabular}{lcccc}
\hline & N & M & D.T. & $\begin{array}{c}\text { Nivel } \\
\text { fiabilidad }\end{array}$ \\
\hline PIP frecuencia & 107 & 3,26 &, 66 &, 92 \\
PIP esfuerzo & 107 & 2,76 &, 82 &, 93 \\
Satisfacción apoyo recibido pareja & 111 & 2,87 &, 94 &, 89 \\
Satisfacción apoyo recibido familia & 111 & 2,77 &, 83 &, 88 \\
Satisfacción apoyo recibido amigos & 111 & 2,60 &, 82 &, 91 \\
Satisfacción apoyo recibido comunidad & 111 & 2,59 &, 84 &, 87 \\
Satisfacción apoyo recibido emocional & 111 & 2,93 &, 66 &, 60 \\
Satisfacción apoyo recibido instrumental & 111 & 2,83 &, 73 &, 67 \\
Satisfacción apoyo recibido informacional & 111 & 2,74 &, 74 &, 62 \\
Satisfacción vital & 110 & 3,53 & 1,17 &, 87 \\
\hline
\end{tabular}

En el siguiente apartado se describen las relaciones existentes entre el estrés percibido por los progenitores, la satisfacción con el apoyo social recibido y la satisfacción vital. Para ello, se han realizado análisis de correlaciones de Pearson, cuyos resultados pueden verse en la tabla 2. Para el estrés percibido se han analizado la frecuencia y el esfuerzo que suponen una serie de actividades relacionadas con el problema médico del hijo enfermo, esto es, las subescalas de PIP frecuencia y PIP esfuerzo. Para la satisfacción con el apoyo social percibido por los padres, se han analizado las cuatro fuentes de apoyo, pareja, familia, amigos y comunidad/asociaciones, así como, los tres tipos de apoyo, emocional, instrumental e informacional.

Respecto al nivel de estrés experimentado por los padres en determinadas situaciones a las que han de enfrentarse durante la enfermedad del hijo, los resultados muestran que existe una correlación negativa y significativa con la satisfacción con el apoyo social percibido por los progenitores. En este caso, se correlacionan negativa y significativamente las dos dimensiones del estrés, frecuencia y esfuerzo, con las cuatro fuentes de apoyo social analizadas (pareja, familia, amigos y asociaciones). Por otro lado, y en cuanto a los tipos de apoyo, se ha encontrado una correlación negativa y significativa con las dos dimensiones del estrés. En concreto, las correlaciones de Pearson han sido significativas y negativas entre la frecuencia y el esfuerzo, y la satisfacción con los tres tipos de apoyo analizados: emocional, instrumental e informacional. En definitiva, el estrés percibido por los padres participantes en el estudio se correlaciona negativa y significativamente con todas las variables que analizan la satisfacción con el apoyo social que perciben los progenitores, tanto a nivel de fuente de apoyo social, como de tipo de apoyo. Por otro lado, el estrés que les provoca a los padres las situaciones relacionadas con su hijo enfermo correlaciona de manera negativa y significativamente con la satisfacción vital. En este caso, la correlación es significativa solo en el caso del PIP esfuerzo, o lo que es lo mismo, el estrés causado por la carga que conllevan determinadas situaciones de la enfermedad del menor. Además, la satisfacción vital también correlaciona positiva y significativamente con la satisfacción con el apoyo recibido de la pareja y con los tres tipos de apoyo social (emocional, instrumental e informacional). 
Tabla 2. Correlaciones del estrés percibido (PIP frecuencia y PIP esfuerzo) con la satisfacción con el apoyo social percibido (fuentes y tipos) y la satisfacción vital

\begin{tabular}{llcc}
\hline & $\begin{array}{c}\text { PIP } \\
\text { frecuencia }\end{array}$ & $\begin{array}{c}\text { PIP } \\
\text { esfuerzo }\end{array}$ & $\begin{array}{c}\text { Satisfacción } \\
\text { vital }\end{array}$ \\
\hline Satisfacción con el apoyo de la pareja &,$- 285(* *)$ &,$- 209(*)$ &, $309(* * *)$ \\
Satisfacción con el apoyo de la familia &,$- 367(* * *)$ &,$- 304(* * *)$ &, 171 \\
Satisfacción con el apoyo de los amigos &,$- 218(*)$ &,$- 365(* * *)$ &, 237 \\
Satisfacción con el apoyo de la comunidad &,$- 235(*)$ &,$- 333(* * *)$ &, 115 \\
Satisfacción con el apoyo emocional &,$- 376(* * *)$ &,$- 358(* * *)$ &, $365(* * *)$ \\
Satisfacción con el apoyo instrumental &,$- 383(* * *)$ &,$- 382(* * *)$ &, $241(*)$ \\
Satisfacción con el apoyo informacional &,$- 294(* * *)$ &,$- 405(* * *)$ &, $297(* * *)$ \\
Satisfacción vital &,- 180 &,$- 316(* * *)$ & 1 \\
\hline
\end{tabular}

$* p<, 05 ; * * p<, 01 ; * * * p<, 001$

Para indagar más sobre la relación existente entre el estrés percibido por los padres que han participado en el estudio, la satisfacción vital y la satisfacción con el apoyo social, se han llevado a cabo una serie de análisis de regresión lineal múltiple. Se pretende examinar si las variables de satisfacción con el apoyo social, en sus diferentes fuentes y tipos, actúan como predictoras del estrés que sufren los progenitores, así como de la satisfacción vital de los mismos. De este modo, por un lado se han realizado análisis de regresión en los que la variable dependiente era la subescala de PIP frecuencia, y en otros la variable dependiente ha sido el esfuerzo. En ambos casos, en los análisis actuaban de variables independientes la satisfacción con el apoyo recibido de las cuatro fuentes de apoyo social y la satisfacción con los distintos tipos de apoyo. Además, también se muestran los análisis de regresión en los que la satisfacción vital actúa como variable dependiente, siendo las variables predictoras todas las relacionadas con las fuentes y tipos de apoyo social.

Los análisis de regresión relacionados con la frecuencia del estrés indican que la variable de apoyo social que predice de manera significativa este estrés percibido por los padres es la satisfacción con el apoyo social recibido de la familia, es decir, la percepción de apoyo familiar disminuye la frecuencia del estrés (tabla 3). La ecuación de regresión tiene una $\mathrm{R}^{2}=0,17, \mathrm{~F}=5,42, \mathrm{p}=0,001$. El resto de las fuentes de apoyo social no han resultado ser estadísticamente significativas. Del mismo modo, también se han realizado análisis de regresión lineal múltiple tomando como variable dependiente la frecuencia del estrés, y como variable independiente los tipos de apoyo. En este caso, es la satisfacción con el apoyo instrumental el tipo de apoyo social que ha resultado estadísticamente significativo en el análisis, es decir, el apoyo instrumental es el que más disminuye la frecuencia del estrés de los progenitores $\left(\mathrm{R}^{2}=0,17, \mathrm{~F}=7,10, \mathrm{p}<0,001\right)$, no encontrándose el apoyo emocional y el informacional como predictores de la frecuencia del estrés en los padres analizados. Estos resultados también pueden consultarse en la tabla 3. 
Tabla 3. Coeficientes estandarizados de regresión de la satisfacción con el apoyo social (fuentes y tipos) sobre la frecuencia del estrés

\begin{tabular}{lrrrrr}
\hline & $\beta$ & $\mathrm{t}$ & $\mathrm{p}$ & $\mathrm{R}^{2}$ & $\mathrm{~F}$ \\
\hline Satisfacción con el apoyo de la pareja &,- 16 & $-1,57$ &, 119 & & \\
Satisfacción con el apoyo de la familia &,- 27 & $-2,65$ &, 009 & & \\
Satisfacción con el apoyo de los amigos &,- 04 &,- 36 &, 720 & & \\
Satisfacción con el apoyo de la comunidad &,,- 10 & $-1,03$ &, 308 & & \\
& & & &, 17 & 5,42 \\
\hline Satisfacción con el apoyo emocional &,- 26 & $-1,69$ &, 093 & & \\
Satisfacción con el apoyo instrumental &,- 32 & $-1,92$ &, 050 & & \\
Satisfacción con el apoyo informacional &,- 17 &,- 998 &, 321 & & \\
& & & &, 17 & 7,10 \\
\hline
\end{tabular}

Variable dependiente: Frecuencia estrés percibido (subescala PIP)

Por otro lado, también se han llevado a cabo análisis de regresión utilizando como variable dependiente la subescala de esfuerzo relacionada con el estrés percibido por los padres, donde la variable independiente era la satisfacción con las distintas fuentes de apoyo social. Se han hallado relaciones estadísticamente significativas con la satisfacción con el apoyo social de amigos y de las asociaciones, es decir, el apoyo de los amigos y de las asociaciones disminuye el esfuerzo relacionado con el estrés. La ecuación de regresión tiene una $\mathrm{R}^{2}=0,21, \mathrm{~F}=6,62, \mathrm{p}<0,001$. No se han encontrado resultados significativos para la satisfacción con el apoyo de familiares y de la pareja (tabla 4). Además, teniendo como variable dependiente el esfuerzo percibido por los progenitores y en relación a la satisfacción con los tipos de apoyo social recibido, los análisis de regresión mostrados en la tabla 4 indican que existe una relación estadísticamente significativa con la satisfacción con el apoyo informacional, en el sentido de que este tipo de apoyo disminuye el estrés relacionado con el esfuerzo que los padres deben realizar. Se ha obtenido la siguiente ecuación de regresión: $\mathrm{R}^{2}=0,17, \mathrm{~F}=7,19, \mathrm{p}<0,001$.

Tabla 4. Coeficientes estandarizados de regresión de la satisfacción con el apoyo social (fuentes y tipos) sobre el esfuerzo relacionado con el estrés

\begin{tabular}{lrrrrr}
\hline & $\beta$ & $\mathrm{t}$ & $\mathrm{p}$ & $\mathrm{R}^{2}$ & $\mathrm{~F}$ \\
\hline Satisfacción con el apoyo de la pareja &,- 03 &,- 36 &, 723 & & \\
Satisfacción con el apoyo de la familia &,- 14 & $-1,35$ &, 181 & & \\
Satisfacción con el apoyo de los amigos &,- 24 & $-2,41$ &, 018 & & \\
Satisfacción con el apoyo de la comunidad &,- 21 & $-2,22$ &, 029 & & \\
& & & &, 21 & 6,62 \\
\hline Satisfacción con el apoyo emocional &,- 07 &,- 48 &, 635 & & \\
Satisfacción con el apoyo instrumental &,- 12 &,- 73 &, 467 & & \\
Satisfacción con el apoyo informacional &,- 41 & $-4,53$ &, 000 & & \\
& & & &, 17 & 7,19 \\
\hline
\end{tabular}

Variable dependiente: Esfuerzo estrés percibido (subescala PIP) 
A continuación, se muestran los resultados de regresión múltiple en los que la satisfacción vital actúa como variable dependiente, siendo la satisfacción con el apoyo social (fuentes y tipos) las variables independientes. De este modo, la satisfacción con el apoyo social de la pareja es la fuente de apoyo que aparece como explicativa de la satisfacción vital $\left(\mathrm{R}^{2}=0,12, \mathrm{~F}=3,61, \mathrm{p}=0,008\right)$, en el sentido de que el apoyo social de la pareja aumenta la satisfacción vital de los progenitores. No se han encontrado resultados significativos con el resto de las fuentes de apoyo social. En el caso de los tipos de apoyo, es la satisfacción con el apoyo emocional la variable que mejor predice la satisfacción vital, esto es, la satisfacción con el apoyo emocional aumenta la satisfacción vital de los padres cuyos hijos están enfermos de cáncer. La ecuación de regresión tiene una $\mathrm{R}^{2}=0,14, \mathrm{~F}=5,83, \mathrm{p}=0,001$. El resto de los tipos de apoyo social no han resultado ser estadísticamente significativos.

Tabla 5. Coeficientes estandarizados de regresión de la satisfacción con el apoyo social (fuentes y tipos) sobre la satisfacción vital

\begin{tabular}{llllll}
\hline & $\beta$ & $\mathrm{t}$ & $\mathrm{p}$ & $\mathrm{R}^{2}$ & $\mathrm{~F}$ \\
\hline Satisfacción con el apoyo de la pareja &, 26 & 2,59 &, 011 & & \\
Satisfacción con el apoyo de la familia &, 02 &, 22 &, 827 & & \\
Satisfacción con el apoyo de los amigos &, 16 & 1,56 &, 123 & & \\
Satisfacción con el apoyo de la comunidad &, 01 &, 09 &, 929 & & \\
& & & &, 12 & 3,61 \\
\hline Satisfacción con el apoyo emocional &, 39 & 2,57 &, 012 & & \\
Satisfacción con el apoyo instrumental &, 16 &, 97 &, 332 & & \\
Satisfacción con el apoyo informacional &, 13 &, 77 &, 442 & & \\
& & & &, 14 & 5,83 \\
\hline
\end{tabular}

Variable dependiente: Satisfacción vital

\section{Discusión y conclusiones}

El análisis multidimensional del apoyo social ha permitido analizar con mayor profundidad la complejidad de las relaciones de apoyo social de los progenitores que acontecen durante la enfermedad oncológica de los hijos. También ha permitido obtener una gran cantidad de información acerca de los lazos de apoyo de los padres con sus principales fuentes y de los tipos de apoyo que más se relacionan con el estrés y la satisfacción vital. Los padres sienten un nivel de estrés medioalto relacionado con el acontecimiento vital de vivir la enfermedad oncológica de un hijo, estando este nivel de estrés más relacionado con el número de veces que tienen que enfrentarse a situaciones difíciles a lo largo de la enfermedad, que con los esfuerzos que tienen que realizar en cada una de esas situaciones (visitas al hospital, sometimiento a diferentes tratamientos, hablar con personal sanitario, hacerse cargo de las necesidades familiares y del hijo enfermo, etc.). Una cuestión importante que arroja estos resultados es que los padres se sienten más estresados con la frecuencia elevada de las situaciones, y no tanto con el esfuerzo. Es interesante tenerlo en cuenta a la hora de ayudar a los padres a poner en marcha estrategias de afrontamiento 
del estrés que les ayuden a reducir la percepción de elevada frecuencia de esas situaciones (ayuda de otros familiares, respiros, organización del tiempo, mejora de las habilidades de comunicación con el personal sanitario, etc.). La satisfacción con el apoyo social en general es media, tanto en la satisfacción que los padres sienten con respecto a las diferentes fuentes de apoyo social (la pareja, la familia, los amigos y la comunidad y asociaciones) como en relación a los diferentes tipos de apoyo que les son proporcionados (apoyo emocional, apoyo instrumental y apoyo informacional). La satisfacción con la vida de los padres de niños enfermos de cáncer también se sitúa en niveles medios.

Un asunto a tener en cuenta que nos muestra este estudio tiene que ver con su perspectiva multidimensional, ya que los resultados obtenidos nos confirman las diferencias que perciben los padres entre las funciones que cumplen las distintas fuentes de apoyo y los tipos de apoyo percibidos. La doble dimensión del estrés (frecuencia y esfuerzo) y las diferentes dimensiones del apoyo social (fuentes y tipos) permiten analizar el contexto de los padres de niños con cáncer de manera más precisa, y este contexto a su vez está formado por distintos sistemas que interactúan y reaccionan de diferente modo ante la enfermedad ${ }^{(11)}$. En relación a las hipótesis planteadas, en general, se han confirmado. Parece clara la relación negativa entre percepción de apoyo social en todas las dimensiones analizadas y el estrés en sus dos dimensiones (frecuencia y esfuerzo), y la relación positiva entre apoyo social y satisfacción vital.

Con respecto a la dimensión tipo de apoyo social (emocional, instrumental e informacional), es el apoyo instrumental el que reduce la percepción de estrés relacionada con el número de ocasiones en las que se tienen que enfrentar a las situaciones estresantes derivadas de la enfermedad del hijo (frecuencia), este apoyo es muy valioso en los casos en los que el estresor es controlable ${ }^{(44)}$, y es el apoyo informacional el que reduce el estrés que sienten ante los esfuerzos que les suponen las situaciones a las que se tienen que enfrentar. Cada tipo de apoyo específico resulta útil para reducir un tipo concreto de estrés, los estudios indican que debe haber una congruencia entre el apoyo recibido y la valoración que hacen las personas sobre su red social y la satisfacción con los recursos que de ella provienen ${ }^{(45)}$. Estos resultados concuerdan con la teoría sobre el apoyo social positivo o funcional, que incide en que, ante situaciones difíciles relacionadas con la salud, la percepción del apoyo es una ayuda positiva y útil cuando reduce el estrés, sin embargo, el apoyo que pueden brindar personas muy cercanas (pareja, familia) si no cumple esta funcionalidad, puede ser percibido como problemático o incluso ser fuente de estrés ${ }^{(46-51)}$. Por tanto, el apoyo social es más beneficioso cuanto más específico es para resolver aquella cuestión por la que se está prestando dicho apoyo ${ }^{(49,51,52)}$. En el estudio se ha comprobado que el apoyo informacional y el apoyo instrumental son efectivos para reducir la percepción de estrés de los padres de niños con cáncer, pero no se relacionan con la satisfacción vital. Únicamente el apoyo emocional es un buen predictor de la satisfacción vital de los padres. Existe un cierto grado de acuerdo en que el apoyo emocional es el más importante para una gran cantidad de problemas ${ }^{(53)}$ pero, sin duda, cada tipo de apoyo cumple con una función específica. Esta información es muy importante de cara a las aplicaciones prácticas que se puedan realizar a la hora de diseñar programas de atención psicosocial a familias de niños con cáncer que tengan en cuenta las necesidades específicas de los padres. Es necesario prestar atención a la red de apoyo familiar de los padres y a las ayudas 
"logísticas" que reciben por parte de ellos, ya que ésta es una cuestión fundamental para aliviar la carga psicológica y física que puede resultar del elevado número de situaciones que tienen que afrontar los padres. Actuaciones como el relevo en los cuidados con otros miembros de la familia, el apoyo en tareas de la nueva rutina de los padres, pueden ayudar a reducir el estrés y mejorar el afrontamiento de la situación $^{(14)}$.

En cuanto a las diferentes fuentes que proporcionan apoyo (pareja, familia, amigos, comunidad/asociación) también han aportado resultados interesantes. El estudio de los diferentes niveles de análisis también queda recogido en los modelos ecológicos ${ }^{(54)}$ que subrayan la importancia del desarrollo de las relaciones sociales de los individuos a través de microsistemas claves, tales como la familia, los amigos y la comunidad. Ha sido importante analizar separadamente el apoyo proporcionado por la pareja, la familia, los amigos y la comunidad ya que cada fuente juega un papel diferente en su relación con el estrés y la satisfacción vital. Los resultados han mostrado que la percepción del apoyo recibido de la familia disminuye la frecuencia del estrés mientras que el apoyo de los amigos y de las asociaciones disminuye el esfuerzo relacionado con el estrés. Es muy interesante que el apoyo de los amigos y la comunidad/asociaciones, en la que se incluyen entidades como la que participa en este estudio, resulte significativo a la hora de reducir el estrés de los padres. Estas dimensiones del apoyo social pueden contribuir a reducir el esfuerzo que suponen estas situaciones para los padres, una correcta información por parte de los agentes implicados en el proceso de la enfermedad y el apoyo de entidades que prestan servicios especializados de atención a las familias juegan un papel muy relevante.

Es interesante destacar el papel del apoyo que procede de la familia ya que ha contribuido a reducir la frecuencia del estrés, algo que no ha ocurrido con el apoyo de la pareja. Una explicación a estos resultados los podemos encontrar en la composición de la muestra del estudio, que lo conforman en su mayoría mujeres tal y como ocurre en muchas de las investigaciones en las que se estudia el papel de las cuidadoras, ya que son las mujeres las que asumen de manera más activa estos roles en nuestra sociedad ${ }^{(55)} \mathrm{y}$, además, participan de manera más significativa en los estudios. En general, las mujeres buscan a otras mujeres como apoyo en los cuidados, son más elegidas como fuentes de apoyo ${ }^{(56,57)}$ y buscan a otras mujeres como confidentes para el afrontamiento de problemáticas de diversa índole ${ }^{(51)}$. Estas mujeres se pueden encontrar en la familia, los amigos y las asociaciones, aunque este aspecto deberá ser analizado con mayor profundidad en otros estudios. Estos resultados son importantes por sus implicaciones prácticas y hay que tenerlos en cuenta ya que el apoyo de la pareja habría que mejorarlo y fomentarlo para reducir el estrés que producen las rutinas diarias en los cuidados del hijo enfermo. Sin embargo, el apoyo de la pareja ha sido el único que se relaciona con el aumento de la satisfacción vital. Una posible explicación es que el apoyo social que ocurre en el entorno más íntimo del individuo es el que más directamente se ha ligado con el bienestar ${ }^{(58)}$.

En conclusión, hay que destacar el papel del apoyo instrumental e informacional en la reducción del estrés, no encontrándose los mismos resultados en cuanto al apoyo emocional, que suele ser el más estudiado ${ }^{(59-64)}$. En cambio, ha sido únicamente el apoyo emocional el que ha contribuido a aumentar la satisfacción vital de los padres. Estos datos nos muestran nuevamente que cada tipo de apoyo cumple una función. La satisfacción con el apoyo viene determinada, en gran parte, por las necesidades de los sujetos $^{(65)}$. Este aspecto queda recogido en la Teoría de la Especificidad de Cohen y 
McKay ${ }^{(52)}$, quienes plantean que el apoyo social será más efectivo cuanto más se ajuste al problema que hay que dar respuesta. Esto explicaría los resultados obtenidos ya que la satisfacción con el apoyo proporcionado por la familia reduce la percepción de estrés ante el número elevado de situaciones a las que tienen que enfrentarse (frecuencia), y el apoyo proporcionado por los amigos y la comunidad/asociaciones reduce el estrés que sienten los padres antes los esfuerzos que deben realizar en dichas situaciones estresantes. Mientras que el apoyo emocional y el apoyo de la pareja son los que más se relacionan con la satisfacción vital de los padres.

Los hallazgos obtenidos en este estudio son muy importantes de cara a su aplicación práctica, conocer el papel de estas variables y sus dimensiones en la adaptación al cáncer pueden contribuir a realizar intervenciones adaptadas a las necesidades psicosociales más relevantes para el manejo de esta situación por parte de los padres ${ }^{(31)}$. Es muy positivo conocer la función que el apoyo social en sus diferentes dimensiones puede tener en los padres de niños con cáncer durante la enfermedad, el impacto positivo que tiene sobre la satisfacción vital de los mismos y la protección que ofrece ante el estrés. Es necesario considerar estas cuestiones en la práctica diaria de los profesionales que prestan apoyo psicosocial en estos casos. Conocer y potenciar las redes de apoyo social y las fuentes y tipos de ayuda a los padres de niños enfermos de cáncer es una de las estrategias terapéuticas que se puede poner en marcha por parte de los profesionales implicados, ya que puede ayudar a mitigar los efectos negativos del estrés, percibir mayor satisfacción vital y, por tanto, mejorar su calidad de vida.

\section{Futuras investigaciones}

De cara a otros estudios sería interesante conseguir una mayor colaboración de padres varones, ya que la participación masculina tiende a ser más baja, y un número de participantes más igualado en sexo puede arrojar resultados que nos permitan conocer las necesidades de apoyo de forma diferencial. En esta línea de investigación sería interesante conocer cómo se relacionan estas variables con otras como la resiliencia, la salud de los padres o la satisfacción familiar, y poder discriminar la muestra por periodos de tiempo desde el diagnóstico del niño, ya que podría haber diferencias en los niveles de estrés, apoyo social y satisfacción vital dependiendo del momento en el que se encuentren los padres dentro del proceso de la enfermedad. También sería interesante llevar a cabo estudios longitudinales con un seguimiento a largo plazo y utilizar a la pareja como unidad de análisis.

\section{Agradecimientos}

A los padres participantes en el estudio y a la Fundación Andrés Olivares.

\section{Referencias bibliográficas}

1. López-Ibor B. Aspectos médicos, psicológicos y sociales del cáncer infantil. Psicooncología 2009; 6: 281-4. [Acceso 1 de febrero de 2019]. Disponible en: $<$ http:// revistas.ucm.es/index.php/PSIC/article/view/PSIC0909220281A> 
2. Die-Trill M. Efectos psicosociales del cáncer en el enfermo pediátrico y su familia. Primeras jornadas internacionales de atención multidisciplinar al niño con cáncer; 1993, 97-114; Valencia: ASPANION.

3. Grau C, Espada MC. Percepciones de los padres de niños enfermos de cáncer sobre los cambios en las relaciones familiares. Psicooncología 2012; 9:125-36. doi: 10.5209/rev_ PSIC. 2012.v9.n1.39142

4. Grau C. La integración escolar del niño con neoplasias. Barcelona: CEAC, 1993.

5. Grau C, Fernández M. Familia y enfermedad pediátrica. An Sist San Navarra 2010; 33(2): 2003-12. [Acceso 1 de febrero de 2019]. Disponible en: http://scielo.isciii.es/scielo. php?script $=$ sci_arttext\&pid=S1137-66272010000300008\&lng=es.

6. Sloper P. Predictors of distress in parents of children with cancer: a prospective study. J Pediatr Psychol 2000; 25: 79-91. doi: 10.1093/jpepsy/25.2.79

7. Norberg A L, Boman K K. Mothers and fathers of children with cancer: loss of control during treatment and posttraumatic stress at later follow-up. Psycho-Oncology 2013; 22: 324-329. doi: 10.1002/pon.2091

8. Vrijmoet-Wiersma C M, Van-Klink J M, Kolk A M, Koopman H M, Ball L M, MaartenEgeler R. Assessement of parental psychological stress in pediatric cancer: a review. J Pediatr Psychol 2008; 33: 694-706. doi: 10.1093/jpepsy/jsn007

9. Gudmundsdottir E, Schirren M, Boman K K. Psychological resilence and long-term distress in Swedish and Icelandic parents'adjustment to childhood cancer. Acta Oncol 2010; 50: 373-80. doi: 10.3109/0284186X.2010.489572

10. Bronfenbrenner U. The ecology of Human Development: experiments by nature and design. Cambridge: Harvard University Press, 1979. Trad. Cast.: La ecología del Desarrollo Humano. Barcelona: Ediciones Paidós, 1987.

11. Bernabeu J, Fournier C, García-Cuenca E, Moran M, Plasencia M, Prades O, et al. Atención interdisciplinar a las secuelas de la enfermedad y/o tratamientos en oncología pediátrica. Psicooncología 2009; 6:381-411. Disponible en: <http://revistas.ucm.es/ index.php/PSIC/article/view/PSIC0909220381A>

12. Torrico E, Santín C, Andrés M, Menéndez S, López M J. El modelo ecológico de Bronfrenbrenner como marco teórico de la Psicooncología. An Psicol 2002; 18: 45-59. Disponible en: https://revistas.um.es/analesps/article/view/28601

13. Grau C. La atención educativa integral del niño enfermo de cáncer en la escuela inclusiva. Acta III Jornadas Internacionales de atención integral al niño con cáncer. Sevilla 1999; 60-8.

14. Espada M C, Grau C. Estrategias de afrontamiento en padres de niños con cáncer. Psicooncología 2012; 9: 25-40. doi: 10.5209/rev_PSIC.2012.v9.n1.39136

15. Gutiérrez-Ajamil E. Arteterapia familiar en oncología pediátrica. Psicooncología 2018; 15:133-51. doi: 10.5209/PSIC.59183

16. Grootenhuis M A, Last B F, Kinderzieken-huis E. Adjustment and coping by parents of children with cancer: A review of literature. Support Care Cancer 1997: 5; 466-88. doi: $10.1007 / \mathrm{s} 005200050116$

17. Patterson J M, Holm K E, Gurney J G. The impact of childhood cancer on the family: A qualitative analysis of strains, resources, and coping behaviors. Psychooncology 2003: 13; 390-407. doi: 10.1002/pon.761

18. Fernández B, Pascual C, Yélamos, C. Cáncer infantil. Guía de apoyo para padres. Madrid: AECC, 2006.

19. Fuemmeler B F, Brown R T, Williams L, Barredo, J. Adjustment of children with cancer and their caregivers: moderating influences of family functioning. Fam Syst Health 2003; 21: 263. doi: 10.1037/1091-7527.21.3.263 
20. Flórez J A. La comprensión del enfermo oncológico. En García-Camba E, editor. Manual de Psicooncología. Toledo: Aula Médica, 1999; p. 171-190.

21. Solomon G F. Psiconeuroinmunología: sinopsis de su historia, evidencia y consecuencias. Comunicación presentada en el $2^{\circ}$ Congreso Virtual de Psiquiatría, Interpsiquis; 2001.

22. Herranz J, Gavilan J. Calidad de vida y cáncer de laringe. Acta Otorrinolaringol Esp 1999; 50: 276-82.

23. Gracia E, Herrero J. Personal and situational determinants of relationship-specific perceptions of social support. Soc Behav Personal 2004; 32: 459-76. doi: 10.2224/ sbp.2004.32.5.459

24. Herrero J, Gracia E. Una medida breve de la sintomatología depresiva (CESD-7). Salud Ment 2007; 30: 40-6.

25. Levitt M J. Social relations in childhood and adolescence: The convoy model perspective. Human Development 2005; 48: 28-47. doi: 10.1159/000083214

26. Hombrados-Mendieta I, Castro-Travé M. Apoyo social, clima social y percepción de conflictos en un contexto educativo intercultural. An Psicol 2013; 29: 108-22. doi: 10.6018/analesps.29.1.123311

27. Gottlieb B H, Bergen A E. Social support concepts and measures. J Psychosom Res 2010; 69: 511-20. doi: 10.1016/j.jpsychores.2009.10.001

28. Taylor S E, Sherman D K, Kim H S, Jarcho J, Takagi K, Dunagan M S. Culture and social support: Who seeks it and why? J Pers Soc Psychol 2004; 87: 354-62. doi: 10.1037/00223514.87.3.354

29. Wills T A, Shinar O. Measuring perceived and received social support. En Cohen S, Underwood L G, Gottlieb B H, editors. Social support measurement and intervention: A guide for health and social scientists. New York: Oxford University Press, 2000; p. 86135. doi: 10.1093/med:psych/9780195126709.003.0004

30. Patterson J M, Holm K E, Gurney J G. The impact of childhood cancer on the family: A qualitative analysis of strains, resources, and coping behaviors. Psychooncology 2004; 13: 390-407. doi: 10.1002/pon.761

31. Choi E K, Yoon S J, Kim J H, Park H J, Kim J Y, Yu E S. Depression and distress in caregivers of children with brain tumors undergoing treatment: psychosocial factors as moderators. Psychooncology 2016; 25: 544-50. doi: 10.1002/pon.3962

32. Hoekstra-Weebers J E, Jaspers J P, Kamps W A, Klip E C. Psychological adaptation and social support of parents of pediatric cancer patients: A prospective longitudinal study. $\mathrm{J}$ Pediatr Psychol 2001; 26: 225-35. doi: 10.1093/jpepsy/26.4.225

33. Musitu G, Buelga S, Lila M, Cava M J. Familia y adolescencia: Análisis de un modelo de intervención psicosocial. Madrid: Síntesis, 2001.

34. Harper F W, Peterson A M, Albrecht T L, Taub J W, Phipps S, Penner, L A. Satisfaction with support versus size of network: Differential effects of social support on psychological distress in parents of pediatric cancer patients. Psychooncology 2016; 25: 551-58. doi: 10.1002/pon.3863

35. Young B, Dixon-Woods M, Findlay M, Heney, D. Parenting in a crisis: conceptualising mothers of children with cancer. Soc Sci Med 2002; 55: 1835-47. doi: 10.1016/S02779536(01)00318-5

36. Grau C. Impacto psicosocial del cáncer infantil en la familia. Educación, Desarrollo y Diversidad 2002; 5: 67-87.

37. Patterson J M, Holm K E, Gurney J G. The impact of childhood cancer on the family: A qualitative analysis of strains, resources, and coping behaviors. Psychooncology 2004; 13: 390-407. doi: 10.1002/pon.761 
38. Gibbins J, Steinhardt K, Beinart H. A systematic review of qualitative studies exploring the experience of parents whose child is diagnosed and treated for cancer. J Pediatr Oncol Nurs 2012; 29: 253-71. doi:10.1177/1043454212452791

39. Manne S, Duhamel K, Redd W H. Association of psychological vulnerability factors to post-traumatic stress symptomatology in mothers of pediatric cancer survivors. Psychooncology 2000; 9: 372-84. doi: 10.1002/1099-1611(200009/10)9:5<372: AIDPON471>3.0.CO;2-1

40. Marsland A L, Long K A, Howe C, Thompson A L, Tersak J, Ewing L J. A pilot trial of a stress management intervention for primary caregivers of children newly diagnosed with cancer: preliminary evidence that perceived social support moderates the psychosocial benefit of intervention. J pediatr psychol 2013; 38(4): 449-61. doi: 10.1093/jpepsy/jss173

41. Del Rincón C, Remor E, Arranz P. Estudio psicométrico preliminar de la versión española del Pediatric Inventory for Parents (PIP). Int J Clin Health Psychol 2007; 7(2): 435-52. Disponible en: http://www.redalyc.org/articulo.oa?id=33717060012

42. García-Martín M A, Hombrados-Mendieta M I, Gomez-Jacinto L. A Multidimensional Approach to Social Support: The Questionnaire on the Frequency of and Satisfaction with Social Support (QFSSS). An Psicol 2016; 32: 501-15. doi: 10.6018/analesps.32.2.201941

43. Diener E D, Emmons R A, Larsen R J, Griffin S. The satisfaction with life scale. J Pers Assess 1985; 49(1): 71-5. doi: 10.1207/s15327752jpa4901_13

44. Cutrona C E, Russell D W. Type of social support and specific stress: Toward a theory of optimal matching. En Sarason B A, Sarason I G, Pierce G R, editors. Social support: An interactional view. New York: Wiley, 1990; p. 319-66.

45. Cohen S, Syme L. Social support and health. New York: Academic Press, 1985.

46. Dunkel-Schetter C, Bennett T L. Differentiating the cognitive and behavioural aspects of social support. En Sarason B A, Sarason I G, Pierce G R, editors. Social support: An interactional view. New York: Wiley, 1990; p. 267-96.

47. Wilcox V L, Kasl S V, Berkman L F. Social support and physical disability in older people after hospitalization: A prospective study. Health Psychol 1994; 13:170-79. doi: 10.1037/0278-6133.13.2.170

48. Abbey A, Abramis D J, Caplan R D. Effects of different sources of social support and social conflict on emotional well-being. Basic Appl Soc Psych 1985; 6:111-19.

49. Revenson T A, Schiaffino K M, Majerovitz S D, Gibofsky A. Social support as a doubleedged sword: The relation of positive and problematic support to depression among rheumatoid arthritis patients. Soc Sci Med 1991; 33: 807-13.

50. Wortman C B, Conway T L. The role of social support in adaptation and recovery from physical illness. En Cohen S, Syme S L, editors. Social support and health. Orlando FI: Academic Press, 1985; p. 281-302.

51. Martos-Méndez M J, Pozo-Muñoz C. Apoyo funcional vs. disfuncional en una muestra de pacientes crónicos. Su incidencia sobre la salud y el cumplimiento terapéutico. An Psicol 2011; 27: 47-57.

52. Cohen S, McKay G. Social support, stressand the buffering hypothesis. A theoretical analysis. En Baum A, Taylor S E, Singer J E, editors. Handbook of Psychology and Health. New Jersey: Hillsdale, 1984; p. 253-67.

53. Cutrona C. Behavioral Manifestations of social support. A microanalytic investigation. J Pers Soc Psychol 1986; 51: 201-08. doi: 10.1037//0022-3514.51.1.201

54. Bronfenbrenner U. Making human beings human: Bioecological perspectives on human development. Thousand Oaks CA: Sage, 2005. 
55. García-Calvente M M, Mateo-Rodríguez I, Maroto-Navarro G. El impacto de cuidar en la salud y la calidad de vida de las mujeres. Gac Sanit 2004; 18(2): 83-92. doi: $10.1157 / 13061998$

56. Hirsch, B J. Psychological dimensions of social networks: A multimethod analysis. Am J Community Psychol 1979; 7 (3): 263-77. doi: /10.1007/BF00890691

57. Burda P C, Vaux A, Shill T. Social support resources: Variation across sex and sex role. Pers Soc Psychol. Bull 1984; 10: 119-26.

58. Kroenke C H, Kubzansky L D, Schernhammer E S, Michelle D, Holmes, M D, Kawachi I. (2006). Las redes sociales, apoyo social, y la supervivencia tras el diagnóstico del Cáncer de Mama. J Clin Oncol 2006; 24: 1105 -1111.

59. Barrera M,Ainlay S L. The structure of social support:A conceptual and empirical analysis. J Community Psychol 1983; 11: 133-43. doi: 10.1002/1520-6629(198304)11:23.0.CO;2-L

60. Cheng C. Getting the right kind of support: Functional differences in the types of social support on depression for Chinese adolescents. J Clin Psychol 1998; 54: 845-49.

61. Chen Y, Feeley TH. Enacted support and well-being: A test of the mediating role of perceived control. Commun Stud 2012; 63(5): 608-25. doi: 10.1080/10510974.2012.674619

62. Harter S. Manual for the social support scale for children. Denver CO: University of Denver, 1985.

63. Malecki C K, Demaray M K. What type of support do they need? Investigating student adjustment as related to emotional, informational, appraisal, and instrumental support. Sch Psychol Q 2003; 18: 231-52. doi: 10.1521/scpq.18.3.231.22576

64. Tardy C H. Social Support Measurement. Am J Community Psychol 1985; 13(2): 187202. doi: 10.1007/BF00905728

65. Lin N. Conceptualizing social support. En Lin N, Dean A, Ensel W, editors. Social support, life events and depression. New York: Academic Press, 1986; p. 17-30. 